\title{
Hipomelanosis de Ito: reporte de caso
}

\author{
Hypomelanosis of Ito: a case report

\section{Mario Alberto Naranjo', Mauricio Torres², Myrna B. Gómez ${ }^{3}$} \\ 1. Médico dermatólogo; docente, Fundación Universitaria de Ciencias Salud, Hospital de San José, Bogotá, D.C., Colombia \\ 2. Médico dermatólogo pediatra; docente, Fundación Universitaria de Ciencias de la Salud; director, programa de Dermatología, \\ Fundación Universitaria de Ciencias de la Salud,, Hospital de San José, Bogotá, D.C., Colombia \\ 3. Médica, residente de Dermatología, Fundación Universitaria de Ciencias de la Salud, Hospital de San José, Bogotá, D.C., Colombia
}

\section{RESUMEN}

La hipomelanosis de Ito es un raro síndrome multisistémico, considerado el tercer trastorno neurocutáneo más frecuente; su incidencia es de 1 por cada 7.540 nacidos vivos en todo el mundo.

Se describe el caso de un adolescente de 12 años con un episodio convulsivo único, asociado a máculas hipocrómicas lineales bilaterales presentes desde el nacimiento, que seguían la distribución de las líneas de Blaschko, localizadas en el tronco y las extremidades.

El diagnóstico de hipomelanosis de Ito se hizo con base en los hallazgos clínicos y en los estudios complementarios.

PALABRAS CLAVE: incontinencia pigmentaria acromática, trastornos de la pigmentación, síndrome de Ito, crisis convulsivas, mosaicismo.

\section{SUMMARY}

Hypomelanosis of Ito (HI) is a rare multisystem syndrome considered the third most common neurocutaneous disorder, with a worldwide incidence of 1 in 7,540 newborns.

We present the case of a male teenager with a history of a single convulsive episode associated with bilateral hypochromic linear macules, following the lines of Blaschko, present at trunk and extremities since birth.

The diagnosis of hipomelanosis of Ito was based on clinical findings and complementary studies results.

KEY WORDS: Incontinentia pigmenti achromians, pigmentation disorders, Ito syndrome, seizures, mosaicism.

\author{
Correspondencia: \\ Mario Alberto Naranjo \\ Email: \\ marionaranjo14@gmail.com \\ Recibido: 27/11/15 \\ Aceptado: 18/o9/18
}

Conflictos de interés:

No se reportan conflictos de interés.

Financiación:

Ninguna. 


\section{INTRODUCCIÓN}

La hipomelanosis de Ito, llamada también incontinencia pigmentaria acrómica o hipomelanosis lineal en bandas estrechas, es un trastorno neurocutáneo poco frecuente, descrito por Ito en 1952. Es la tercera enfermedad más frecuente entre los síndromes neurocutáneos, después de la neurofibromatosis de tipo 1 y la esclerosis tuberosa ${ }^{(1)}$. El tipo de herencia no está claramente definido, aunque en la mayoría de las revisiones lo asocian a un 'mosaicismo' cromosómico, especialmente de los genes 9q33-qter, 15q11-q13 у Xp11; menos del 3 \% de los casos presentan un patrón de herencia autosómica dominante (2).

La incidencia global es de 1 por cada 7.540 nacidos vivos, con prevalencia de 1 por cada 82.000 individuos, y predominio del sexo femenino con una proporción de 2 a ${ }^{1}{ }^{(3)}$. En Colombia, existe un reporte de dos casos en el departamento de Santander, pero se desconocen las características clínicas de estos pacientes.

Clínicamente, se presentan manifestaciones cutáneas y extracutáneas; las cutáneas, presentes en el $100 \%$ de los casos, se caracterizan por la aparición de máculas hipopigmentadas distribuidas de forma lineal o anular, siguiendo las líneas de Blaschko; se encuentran presentes desde el nacimiento en el $50 \%$ de los casos y, en el resto, aparecen durante los primeros meses de vida después del primer bronceado ${ }^{(4)}$.

Las manifestaciones extracutáneas incluyen alteraciones neurológicas, músculo-esqueléticas (escoliosis, deformidad torácica y anormalidades en los dedos), ováricas, oculares y renales; de estas, las alteraciones neurológicas son las de mayor relevancia clínica, están presentes en 76 a $94 \%$ de los pacientes, e incluyen discapacidad intelectual, retraso en el desarrollo psicomotor y epilepsia ${ }^{(5,6)}$.

Estas manifestaciones hacen parte de los criterios diagnósticos descritos por Ruiz-Maldonado en 1992 (tabla 1), los cuales actualmente son poco utilizados ya que, para el diagnóstico clínico, se tienen en cuenta la hipopigmentación característica de esta anomalía y las manifestaciones extracutáneas ${ }^{(4,7)}$.

\section{PRESENTACIÓN DEL CASO}

Se presenta el caso de un menor de sexo masculino, de 12 años de edad, que presentaba desde el nacimiento máculas hipopigmentadas con patrón de distribución blaschkoide, asintomáticas, localizadas en el abdomen, que posteriormente se generalizaron y comprometieron casi toda la superficie corporal (figuras 1, 2 y 3). Tenía como antecedente un episodio convulsivo único a los cinco años de edad, tratado por neurología

Tabla 1. Criterios diagnósticos de la hipomelanosis de Ito, propuestos por Ruiz-Maldonado (1992)

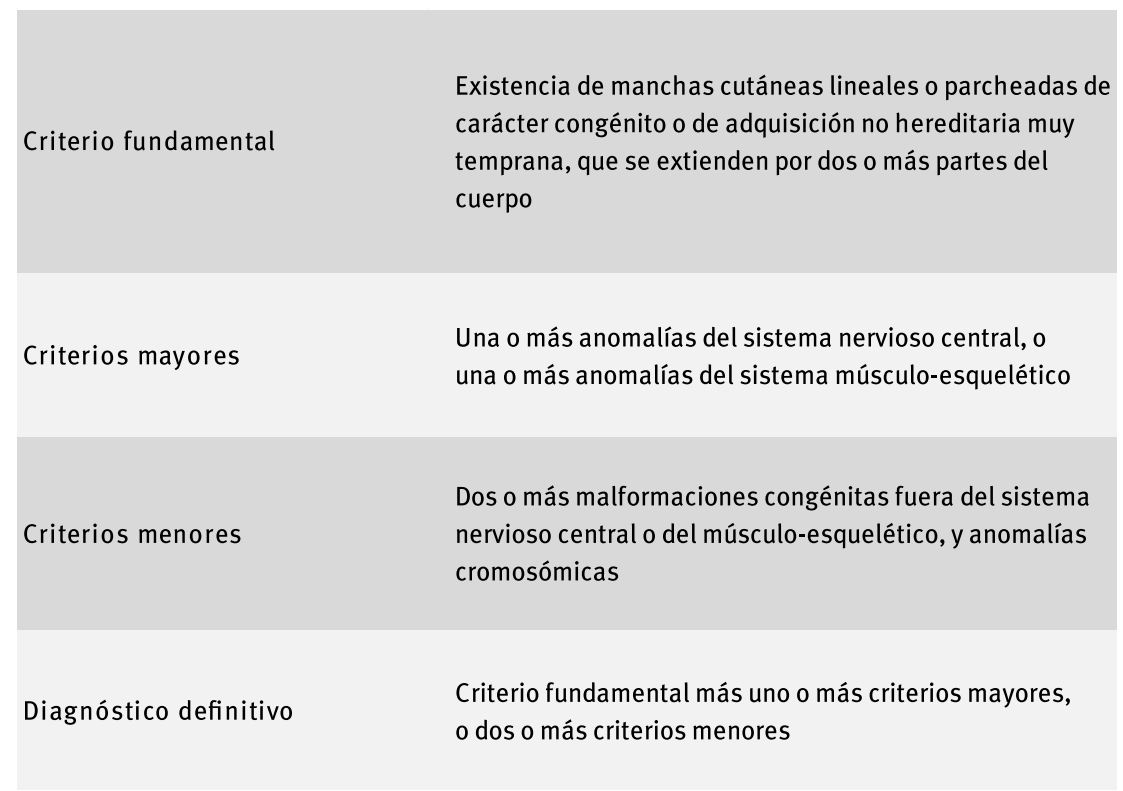




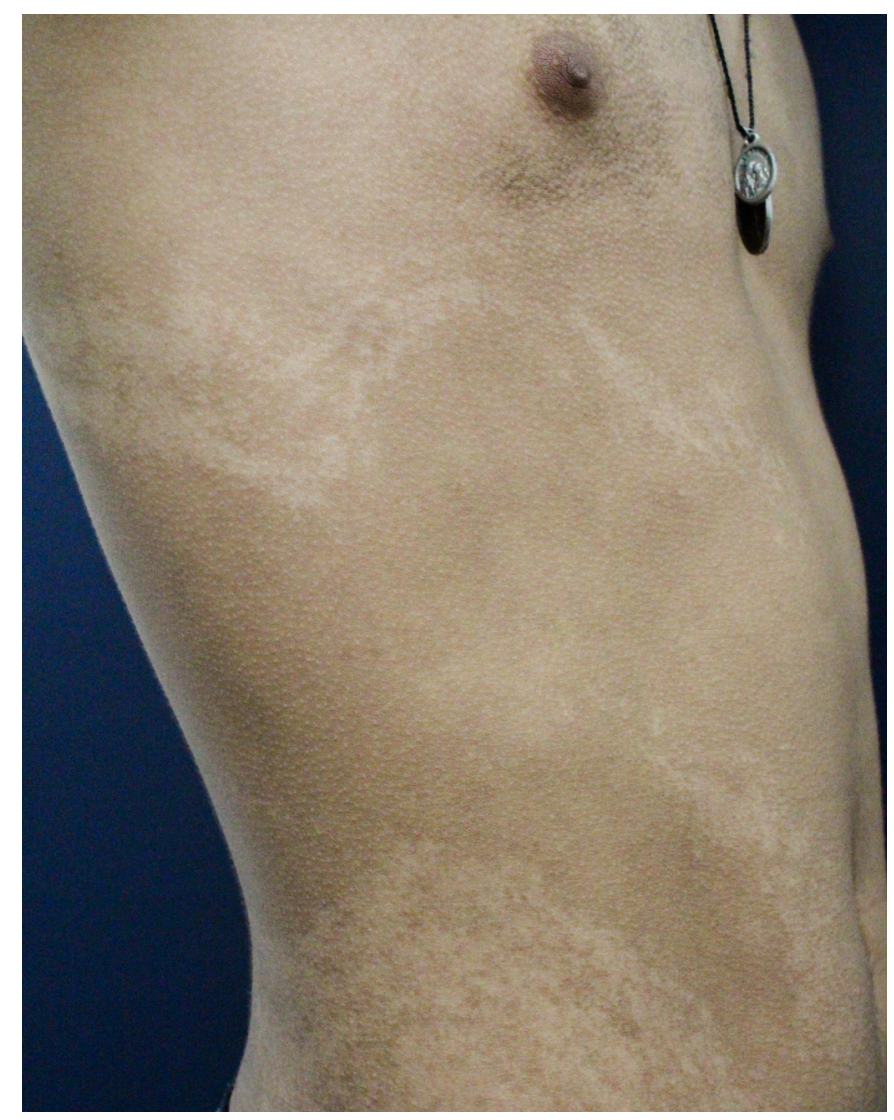

Figura 1. Máculas hipocrómicas de distribución blaschkoide en la cara lateral de abdomen y tronco

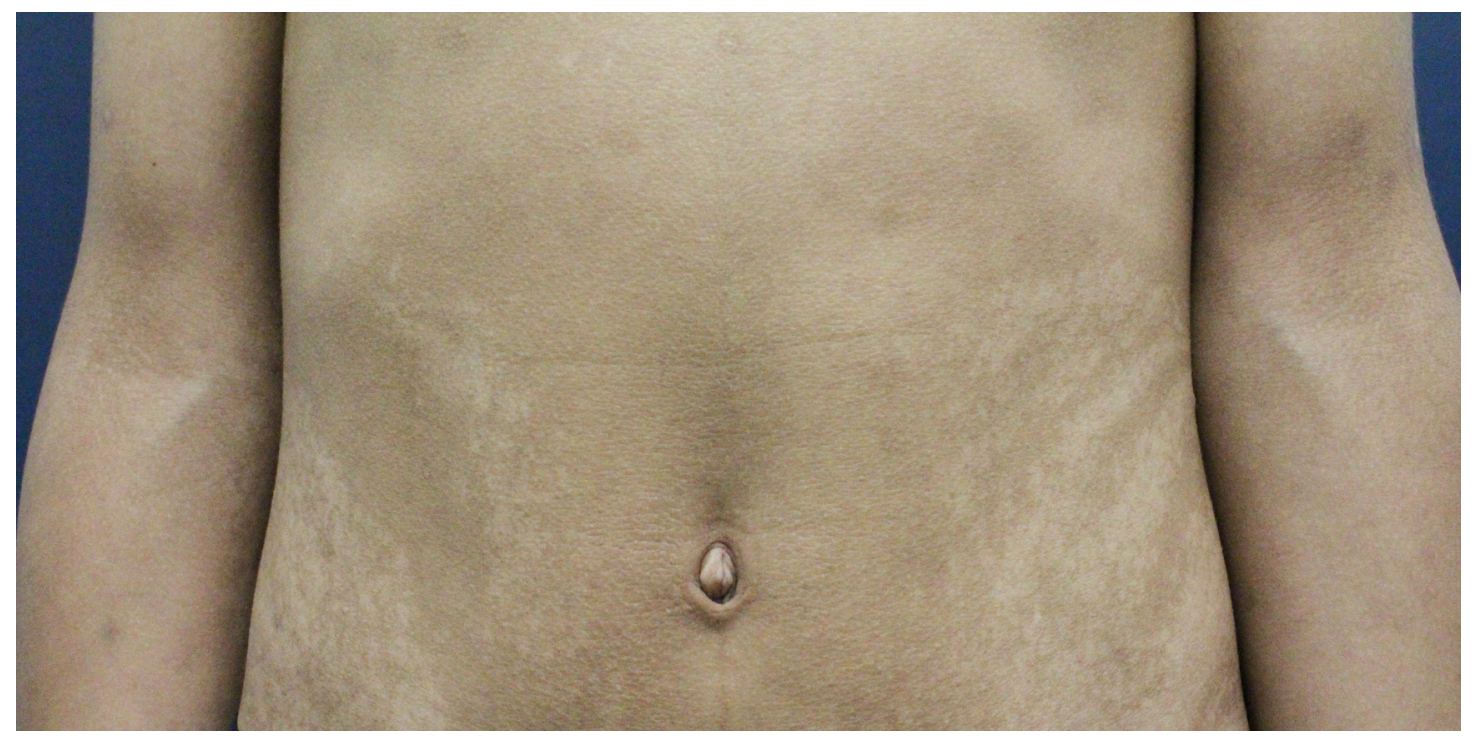

Figura 2. Máculas hipocrómicas de distribución blaschkoide en la cara anterior de abdomen y antebrazo 


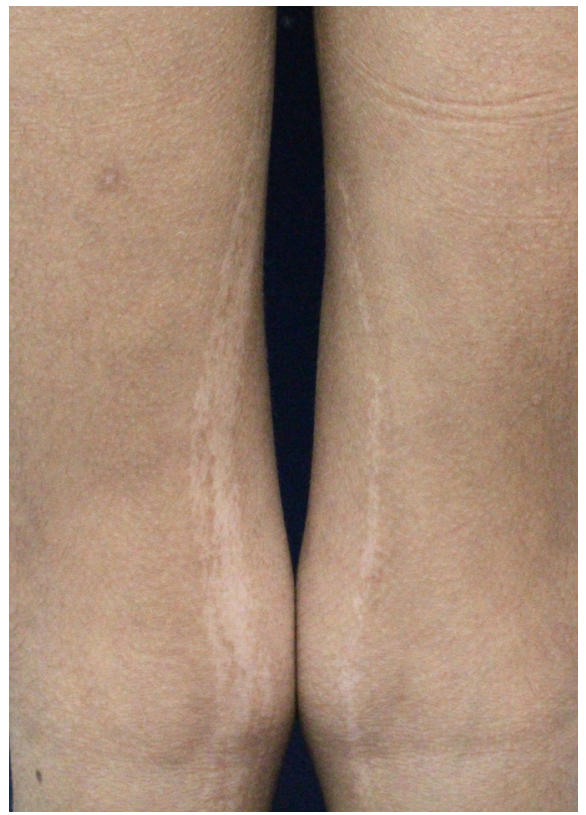

Figura 3. Máculas hipocrómicas de distribución blaschkoide en las extremidades inferiores

pediátrica, actualmente asintomático. No refirió antecedentes familiares de importancia.

En estudios de extensión, presentó cariotipo 46XY, 9qh-, una metafase con disminución heterotrófica de un cromosoma 9. Los estudios músculo-esqueléticos evidenciaron una escoliosis leve, y los estudios imagenológicos de los sistemas cardiovascular y renal fueron normales.

\section{DISCUSIÓN}

Existen numerosas enfermedades genéticas que se manifiestan por medio de la piel; una de ellas es el 'mosaicismo', el cual se caracteriza por la presencia de dos o más poblaciones de células genéticamente distintas (8). La hipomelanosis de ito forma parte de los 'mosaicismos' neurocutáneos, los cuales se caracterizan por lesiones hipopigmentadas que siguen una distribución blaschkoide y se presenta secundario a la interrupción en la producción de melanina durante la melanogénesis, que pueden estar presentes desde el nacimiento o presentarse en la primera infancia; estas manifestaciones cutáneas están presentes en el $100 \%$ de los $\operatorname{casos}^{(4,6)}$.

Además, este síndrome está asociado a afecciones extracutáneas, entre las que se encuentran, en orden de frecuencia, alteraciones del sistema nervioso central (90 \%) y alteraciones músculo-esqueléticas $\left(70 \%{ }^{(6)}\right.$. El caso que reportamos presentó un episodio convulsivo único a los cinco años de edad y afectación esquelética por escoliosis leve.

Estos hallazgos clínicos permiten el diagnóstico definitivo de hipomelanosis de Ito. Aunque en la histopatología se observa disminución de la melanina en la piel hipopigmentada con un número de melanocitos normal y disminuidos al contrastarlo con la piel normal, estos hallazgos histopatológicos son inespecíficos ${ }^{(9)}$. Entre las teorías asociadas a la alteración genética responsable de esta enfermedad, se han descrito alteraciones autosómicas y algunas ligadas a la traslocación o inactivación del cromosoma $\mathrm{x}^{(10)}$. Es importante resaltar que el presente paciente tiene un cariotipo con una metafase con disminución heterotrófica de un cromosoma 9, una alteración genética que no ha sido descrita dentro del grupo clásico de asociaciones.

\section{CONCLUSIÓN}

Se presenta el caso de un paciente con hipomelanosis de Ito, el cual es un trastorno neurocutáneo poco frecuente, secundario a un 'mosaicismo' genético. Es importante que, como dermatólogos, conozcamos las 
manifestaciones clínicas cutáneas y las extracutáneas de esta enfermedad, las cuales la diferencian de otras alteraciones hipopigmentarias que siguen una distribución blaschkoide.

El diagnóstico se hace por medio de los hallazgos clínicos, cutáneos, neurológicos y músculo-esqueléticos, ya que no es posible confirmar el diagnóstico genético debido a la heterogeneidad de esta entidad; por lo tanto, se requiere un enfoque multidisciplinario para el tratamiento oportuno de trastornos que pueden afectar la funcionalidad del paciente.

\section{AGRADECIMIENTOS}

A Mónica Novoa, Édgar Olmos y Óscar Mora, por su valiosa colaboración.

\section{REFERENCIAS}

1. Gómez-Lado C1, Eirís-Puñal J, Blanco-Barca O, del Río-Latorre E, Fernández-Redondo V, CastroGago M. Hypomelanosis of Ito. A possibly underdiagnosed heterogeneous neurocutaneous syndrome. PubMed - NCBI. Rev Neurol. 2004 Feb 1-15;38(3):223-8.

2. Ruggieri M, Pavone L. Hypomelanosis of Ito: Clinical syndrome or just phenotype? J Child Neurol. 20 J Child Neurol. 200o Oct;15(10):635-44

3. Park JM, Kim HJ, Kim T, Chae HW, Kim DH, Lee MG. Sexual precocity in hypomelanosis of Ito: Mosaicism-associated case report and literature review. Int J Dermatol. 2011;50:168-74.

4. Romero A, Salazar M, Tufino M, Villacís A, Galarza F. Hipomelanosis de Ito. Dermatol Rev Mex. 2015;59:43-8.

5. Pascual-Castroviejo I, Roche C, Martínez-Bermejo A, Arcas J, López-Martín V, Tendero A, et al. Hypomelanosis of Ito. A study of 76 infantile cases. Brain Dev. 1998;20:36-43.

6. Ream M. Hypomelanosis of Ito. Handb Clin Neurol. 2015;132:281-9.

7. Ruiz-Maldonado R, Toussaint S, Tamayo L, Laterza A, del Castillo V. Hypomelanosis of Ito: Diagnostic criteria and report of 41 cases. Pediatr Dermatol. 1992;9:1-10.

8. Kouzak SS, Mendes MS, Costa IM. Cutaneous mosaicisms: Concepts, patterns and classifications. An Bras Dermatol. 2013;88:507-17.

9. Pavone V, Signorelli SS, Praticò AD, Corsello G, Savasta S, Falsaperla R, et al. Total hemi-overgrowth in pigmentary mosaicism of the (hypome- lanosis of) Ito type: Eight case reports. Medicine (Baltimore). 2016;95:e2705.

10. Taibjee SM, Bennett DC, Moss C. Abnormal pigmentation in hypomelanosis of Ito and pigmentary mosaicism: The role of pigmentary genes. $\mathrm{Br} \mathrm{J}$ Dermatol. 2004;151:269-82. 